\title{
Searching for signatures of cold adaptation in human TRP genes
}

\author{
A.V. Igoshin ${ }^{1 *}$, K.V. Gunbin ${ }^{1}$, N.S. Yudin ${ }^{1,2}$, M.I. Voevoda ${ }^{1,2,3}$ \\ ${ }^{1}$ Institute of Cytology and Genetics SB RAS, Novosibirsk, Russia \\ ${ }^{2}$ Novosibirsk State University, Novosibirsk, Russia \\ ${ }^{3}$ Institute of Internal and Preventive Medicine - branch of ICG SB RAS, Novosibirsk, Russia \\ *e-mail:igoshin@bionet.nsc.ru
}

Key words: cold adaptation, TRP genes, minimum winter temperature, SNPs, human population

Motivation and Aim: Since his origin from sub-Saharan Africa 200,000 years ago, modern human eventually spread across the Earth. These migrations of Homo sapiens have led to adaptation to local environments. The advent of high-throughput next-generation sequencing and microarray technologies has enabled researchers to discover genetic bases of such adaptations. Here, applying three different statistical approaches, we try to detect signatures of cold adaptation in seven human TRP genes (TRPA1, TRPM8, TRPV1, TRPV2, TRPV3, $T R P V 4$, and TRPM3) as being immediately involved in thermoregulation in mammals.

Methods and Algorithms: 407 SNP IDs were obtained from dbSNP database by querying gene's names. Information about minimum winter temperature was taken from NCEPNCAR database using coordinates from study by H. Cann et al. [1]. Genotypic data was taken from HGDP database, and then imputed with fastPhase software. Bayesian linear model (BLM) implemented in Bayenv2 software accounts for sample size and covariance of allele frequencies across populations, but not robust to outliers and less sensitive to nonlinear relationships. Spearman's rank correlation test (SRC) from Bayenv2 uses allele frequencies standardized to have no covariance. It is less powerful than BLM, but more robust to outliers and can detect nonlinear relationships. BayScenv approach associates $F_{\text {st }}$ distances with an environmental variable, but does not account for covariance. For BLM, covariance matrix was obtained by running 100,000 iterations of Markov Chain Monte Carlo (MCMC) algorithm and averaging across 20 matrix estimates. Then, for each SNP we ran 200,000 MCMC iterations. Because of relatively low reproducibility of Bayenv2, we repeated this procedure 5 times. Bayesian factor averaged across all repetitions was used as a final estimate for each SNP. The same rules were also used for SRC. BayScenv model was run with default options except that parameter $p$ was set to 0.25 . Two repetitions were done. Results: At the intersection of top $1 \%$ of all three methods, we found SNP rs 17617922 , in TRPM3 gene that has already been reported to be associated with minimum winter temperature among human populations by whole genome scan [2]. This SNP does not have clear evidences supporting its functional effect, so it is possible that rs17617922 is linked to some causal variant. In addition, we found SNP rs8065080 in TRPV1 gene being at the top $1 \%$ of SRC and BLM results. It is interesting that this SNP leads to amino acid substitution. Furthermore, individuals homozygous for allele present at high frequency in regions with cold climate have higher tolerance to cold [3].

Conclusion: SNPs rs17617922 in TRPM3 gene and rs8065080 in TRPV1 gene are probably associated with human adaptation to cold climate. Further studies based on detecting selective sweeps are needed.

\section{References}

1. Cann H. et al. (2002) A human genome diversity cell line panel. Science. 296(5566):261-262.

2. Günther T., Coop G.(2013) Robust identification of local adaptation from allele frequencies. Genetics. 195:205-220.

3. Binder A. et al. (2011) Transient receptor potential channel polymorphisms are associated with the somatosensory function in neuropathic pain patients. PLoS One. 6:e17387. 